\title{
Revisión
}

\section{Hiperaldosteronismo primario, una nueva perspectiva}

\author{
Karen Gómez- Hernández, Chih Hao Chen-Ku
}

\section{Resumen}

Hace cincuenta años se describió por primera vez el hiperaldosteronismo primario que hasta hace poco se consideraba un síndrome infrecuente y una causa excepcional de hipertensión arterial. De cada 10 pacientes hipertensos habrá en promedio uno de ellos con este padecimiento, convirtiendo a tal grupo de enfermedades en la causa más frecuente de hipertensión potencialmente curable. Este incremento en la prevalencia se debe quizás al aumento en la sensibilidad de las pruebas de tamizaje utilizadas. Si bien es cierto, los nuevos hallazgos no implican necesariamente el tamizaje generalizado de la población de hipertensos; es importante que el médico que la controla esté familiarizado con el tema para que lo considere y lo sospeche en el manejo y seguimiento de sus pacientes.

Descriptores: Hiperaldosteronismo primario, hipertensión arterial, prevalencia

Key words: primary hyperaldosteronism, hypertension, prevalence

Recibido: 28 de noviembre de 2005

Aceptado: 22 de agosto de 2006

\section{Historia}

En 1563, Bartolomeo Eustacchio fue quien hizo la primera mención bibliográfica de la glándula adrenal. ${ }^{1}$ Siglos después, en 1855, Thomas Addison describió el primer síndrome clínico asociado a esta glándula: insuficiencia suprarrenal. ${ }^{2,3}$ Este descubrimiento impulsó la investigación de la glándula adrenal. El enfoque durante los primeros años del S. XX fue el de dilucidar el papel de los glucocorticoides. Al inicio se pensó que éstos eran los responsables de

Servicio de Endocrinología, Hospital San Juan de Dios, Caja Costarricense de Seguro Social. Abreviaturas:

AP, hiperaldosteronismo primario;APA adenoma productor de aldosterona; ARR, relación de concentración de aldosterona plasmática a actividad de renina plasmática; ATP adenosíntrifosfato; $\mathrm{HU}$, hiperplasia unilateral; IHA, hiperplasia bilateral idiomática; PAC concentración de aldosterona plasmática; PRA actividad de renina plasmática; UNAM, ácido ribonucléico mensajero.

Correspondencia: Karen Gómez Hernández Email: karengomher@yahoo.es

ISSN 0001-6002/2007/49/1/11-18 Acta Médica Costarricense, (C2007 Colegio de Médicos y Cirujanos la mayoría de la actividad mineralocorticoide, por no contar con un ensayo lo suficientemente sensible para detectarla. ${ }^{1}$ A principios de la década de los años cincuenta del siglo pasado, Simpson y Tait desarrollaron un bioensayo de alta sensibilidad para la actividad mineralocorticoide y luego reportaron la estructura de la aldosterona. ${ }^{1,4}$ Poco después, en 1954, una paciente de 34 años, M.W., consultó al hospital universitario en donde se encontraba trabajando Jerome W. Conn. La paciente aquejaba una historia de 7 años de evolución de debilidad muscular episódica que usualmente resultaba en parálisis de las piernas y espasmos musculares. Además, refería poliuria, nocturia, hipertensión sin signos de hipercortisolismo, hipernatremia, hipokalemia y alcalosis metabólica. Esta presentación clínica hizo que Conn sospechara que la paciente tenía exceso de hormona mineralocorticoide (probablemente aldosterona) y durante los siguientes siete meses la estudió en la Unidad de Investigación Metabólica. El Dr. Conn indicó sus hallazgos en el discurso presidencial a la Sociedad Central para la Investigación Clínica y describió así el síndrome que llamó aldosteronismo primario, diciendo que "una adrenalectomía total seguida de tratamiento de sustitución aboliría toda la anormalidad metabólica". 5 En 1955 se comprobó la hipótesis de Conn, ya que se le hizo exploración quirúrgica de las glándulas adrenales a M.W. y se encontró un tumor de $4 \mathrm{~cm}$. 


\section{Definición y clasificación}

El síndrome de hiperaldosteronismo primario se refiere a un grupo de trastornos en los cuales la producción excesiva de aldosterona, en la zona glomerulosa de la corteza adrenal, ocurre de forma independiente(oparcialmente independiente) de la estimulación normal. ${ }^{6-8}$ Esta secreción excesiva de hormona mineralocorticoide produce hipertensión arterial y se acompaña de supresión de renina plasmática, expansión del sodio corporal total y tendencia a la hipokalemia. ${ }^{9}$ Hasta elmomentohay identificadas 5 formas dehiperaldosteronismo primario (Cuadro 1). ${ }^{10}$ La bibliografía no es uniforme en cuanto a su frecuencia. Algunos autores establecen que el aldosteronoma es la causa más común y representa entre un $50 \%$ y un $60 \%$ de los casos de hiperaldosteronismo primario. ${ }^{11-13}$ Sin embargo, probable-mente debido al uso más generalizado de pruebas de tamizaje, la hiperplasia bilateral idiopática constituye o llegue a constituir la causa más frecuente. ${ }^{10,14}$ A pesar de la controversia, en definitiva estas 2 causas juntas son responsables de la gran mayoría $(>90 \%)$ de los casos de aldosteronismo primario. ${ }^{6,10,12,15,16}$

\section{Prevalencia}

Hasta hace algunos años se creía que la prevalencia de este síndrome era $<2 \%$ en pacientes no seleccionados con hipertensión. ${ }^{7,17-19}$ Actualmente se estima que la prevalencia real es de un promedio del $10 \% .{ }^{20}$ Este aumento en el número de casos ha sido ampliamente demostrado en diferentes países del mundo en una serie de estudios recientes (Gráfico 1). ${ }^{21-31}$ No existe duda de que el hiperaldosteronismo primario es la causa más frecuente de hipertensión secundaria. Sin embargo, algunos autores no coinciden con que haya un aumento tan grande en la prevalencia y que los datos deben interpretarse con cautela, ya que los diferentes estudios de prevalencia han utilizado métodos de tamizaje de diagnóstico distintos. $^{32}$ Otros plantean que una proporción de los individuos que se están catalogando dentro del síndrome de hiperaldosteronismo primario, en realidad presentan hallazgos de actividad de renina plasmática compatibles con la evolución natural de la hipertensión arterial o hipertensión arterial esencial hiporreninémica. ${ }^{33,34}$

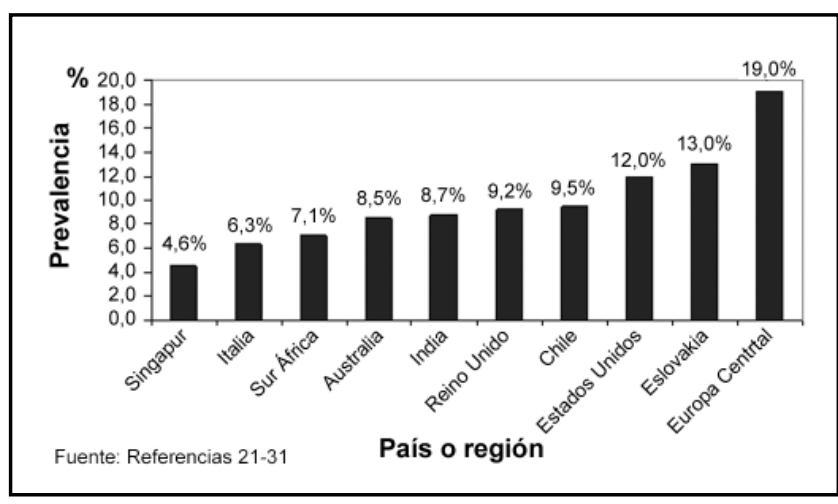

Figura 1. Prevalencia de hiperaldosteronismo primario en pacientes hipertensos en diferentes países y regiones

\begin{tabular}{l}
$\begin{array}{l}\text { Cuadro 1. Formas de hiperaldosteronismo } \\
\text { primario }\end{array}$ \\
Adenoma productor de aldosterona \\
Hiperplasia bilateral idiopática \\
Hiperplasia adrenal primaria (unilateral) \\
Carcinoma adrenocortical productor de aldosterona \\
Hiperaldosteronismo familiar \\
Aldosteronismo remediable con glucorticoides \\
Hiperaldosteronismo familiar tipo II \\
\hline Modificado de: Endocrinology 2003; 144(6): 2208-2213 \\
\hline
\end{tabular}

\section{Fisiología y fisiopatología}

La aldosterona es una hormona esteroidal sintetizada a partir de colesterol en la zona glomerulosa de la corteza adrenal, en respuesta a estímulos que promueven su secreción. ${ }^{35}$ Estos estímulos son: angiotensina II, aumento en la concentración de potasio plasmático, adrenocorticotropina, niveles disminuidos de sodio plasmático y de factor natriurético atrial. ${ }^{36}$ De todos estos factores el más importante es la renina, y normalmente existe una relación casi lineal entre la actividad de renina plasmática y la secreción urinaria de aldosterona. ${ }^{35-37} \mathrm{~A}$ su vez, la secreción de renina desde el aparato yuxtaglomerular renal se da en respuesta a perfusión disminuida, disminución en la ingesta de sodio y estimulación simpática. ${ }^{38}$ La renina es responsable de convertir el angiotensinógeno a angiotensina $\mathrm{I}$, que rápidamente es convertida a angiotensina II en los pulmones, por la enzima convertidora de angiotensina. ${ }^{39}$

Las funciones principales de la aldosterona son: mantener el volumen del líquido extracelular mediante la conservación de sodio corporal e impedir la sobrecarga de potasio. ${ }^{40,41}$ Esto lo logra mediante la unión a su receptor citosólico, con el cual forma un complejo que luego es traslocado al núcleo en donde activa la transcripción de RNAm. Los productos proteicos se encargan de aumentar el transporte de sodio con el aumento de la permeabilidad capilar de la membrana apical al sodio, aumento de la ATPasa de $\mathrm{Na}^{+} / \mathrm{K}^{+}$en las membranas basolaterales, y de la actividad de las enzimas del ciclo del ácido cítrico que catalizan la formación de $\mathrm{ATP}^{42}$

En el hiperaldosteronismo primario la producción excesiva de aldosterona tiene varias características importantes que se deben entender para comprender e interpretar de manera adecuada las pruebas diagnósticas y de tamizaje. Primero, la secreción de aldosterona no responde necesariamente ante los efectos inhibitorios de niveles bajos de potasio sérico, expansión de volumen ni ingesta de sodio aumentada. ${ }^{7,43,44}$ Segundo, al igual que los otros trastornos primarios de exceso de mineralocorticoides, se asocia a supresión de la renina plasmática. ${ }^{35}$ Tercero, es parcialmente autónoma y está influenciada de forma importante por 
corticotropina en la mayoría de casos de aldosteronoma. ${ }^{7}$ Cuarto, en gran parte de los pacientes se ha perdido su respuesta normal a la angiotensina II (en un 20\% de los pacientes con aldosteronoma $y$ en los pacientes con hiperplasia bilateral sí hay respuesta, y por lo tanto, la respuesta de la concentración plasmática de aldosterona con los cambios de postura es normal). ${ }^{7}$ Quinto, en el caso del aldosteronismo remediable con glucocorticoides, es regulada por adrenocorticotropina. ${ }^{45-47}$ Esto se debe a que la enfermedad autosómica dominante es causada por el cruzamiento desigual entre los genes CYP11B1 y CYP11B2, lo que resulta en un gen quimérico con actividad sintetasa de aldosteronatotalmentedependientedeadrenocorticotropina. ${ }^{46}$ Esta es una enfermedad extrema-damente rara y la prevalencia no es conocida.

Se puede concluir que la hiperplasia bilateral idiopática se comporta fisiopatológicamente como una exageración de lo normal, secretando grandes cantidades de aldosterona en respuesta a angiotensina II desde ambas glándulas. También, que hay 2 tipos de aldosteronoma, los que responden a corticotropina y los que responden a angiotensina II. El aldosteronoma y la hiperplasia unilateral se comportan de manera muy similar.

Las consecuencias fisiopatológicas del exceso de aldosterona son múltiples. En un inicio la hipertensión es secundaria a retención de sodio (sin presencia de edema), sin embargo, con el paso del tiempo hay un escape de este mecanismo. ${ }^{8}$ De forma crónica, el aumento de la resistencia periférica total secundaria a una alteración en el metabolismo del calcio por exceso intracelular de sodio, es responsable de mantener la presión arterial elevada. ${ }^{8,48}$ Existen también alteraciones en el medio interno que incluyen hipokalemia, hipercalciuria y alcalosis metabólica (Figura 2). ${ }^{36}$ Recientemente, se ha relacionado el exceso de aldosterona con enfermedad cerebrovascular, fibrosis cardiaca, hipertrofia ventricular e inflamación vascular. ${ }^{49-54}$

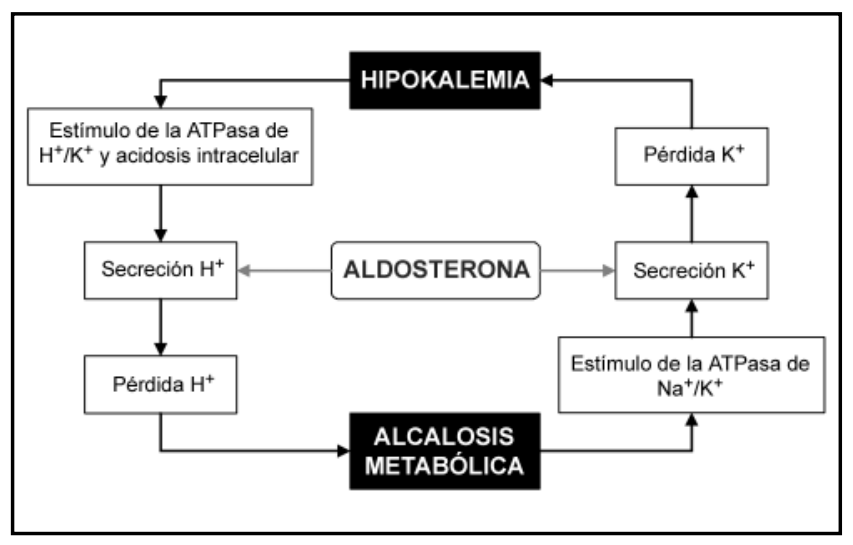

Figura 2. Relación de retrocontrol positivo entre alcalosis metabólica e hipokalemia y efecto del exceso de aldosterona

\section{Cuadro clínico}

La presentación clínica clásica de hiperaldosteronismo primario implica la presencia de hipertensión arterial aunada a los hallazgos de hipokalemia y alcalosis metabólica. ${ }^{5}$ Pero esta presentación del síndrome no siempre se produce, ya que muchos individuos cursan con normokalemia. ${ }^{57}$ Además, un porcentaje importante de los afectados son asintomáticos. Cuando aparecen síntomas, probablemente sean secundarios a la hipertensión y la hipokalemia. ${ }^{7}$ La hipertensión del hiperaldosteronismo primario suele ser más severa y su manejo requiere generalmente múltiples medicamentos, sin lograr obtener una respuesta adecuada. ${ }^{48}$ En el caso de la hipokalemia, muchas veces se hace evidente hasta que se utilicen diuréticos y puede ser una de las claves del diagnóstico. Los síntomas relacionados con esta dependen tanto de la velocidad de instauración como de su severidad. ${ }^{58}$ Con hipokalemia moderada aparecen síntomas inespecíficos, como debilidad generalizada, estreñimiento y lasitud. ${ }^{58} \mathrm{La}$ hiperkalemia severa $(<2.5 \mathrm{mmol} / \mathrm{l})$ puede asociarse a necrosis muscular y parálisis ascendente. ${ }^{58}$

Existen características distintivas en cada uno de los subgrupos de hiperaldosteronismo primario. El adenoma adrenal generalmente es $<2 \mathrm{~cm}$ de diámetro, se localiza más en la glándula izquierda que en la derecha. ${ }^{59}$ Afecta con mayor frecuencia a mujeres que a hombres durante la cuarta y quinta década de vida, ocasionalmente puede afectar a mujeres más jóvenes. ${ }^{7,59}$ Debido a que expresa la vía de la C-18 oxidación, la cual no está presente en individuos con hiperplasia bilateral, en la orina de estos pacientes se pueden encontrar 18-hidroxicortisol y 18-oxocortisol. ${ }^{60}$

La hiperplasia idiopática bilateral se caracteriza por hiperplasia difusa y focal entremezclada con pequeños nódulos córticosuprarrenales. ${ }^{59}$ Es predominante en los hombres y generalmente se presenta a edades más tardías que el aldosteronoma. $^{7}$

El aldosteronismo remediable por glucocorticoides tiene como particularidad que la hipertensión es usualmente de inicio temprano en la vida y que puede ser de suficiente severidad como para causar muerte temprana, comúnmente asociada a hemorragia intracerebral. ${ }^{61}$

El carcinoma corticosuprarrenal es extremadamente raro y se puede encontrar a cualquier edad. ${ }^{59}$ Suelen ser tumores muy malignos que cuando se descubren tienen gran tamaño. ${ }^{59}$ Cuando estos son funcionales, se presentan como tumores virilizantes y no como productores de aldosterona, por lo que constituyen una causa muy rara de hiperaldosteronismo.

\section{Tamizaje y diagnóstico}

En 1981 se propuso por primera vez el uso de la relación de la concentración de aldosterona plasmática con la actividad de renina plasmática como método de tamizaje ${ }^{62} \mathrm{y}$ es actualmente el método de elección para este fin., ${ }^{7,63,64} \mathrm{Su}$ 
uso es lo que justifica el aumento en el diagnóstico de la enfermedad en la actualidad. ${ }^{65,66}$ Existen ya establecidos varios criterios clínicos para el tamizaje (Cuadro 2). ${ }^{10,67} \mathrm{~A}$ pesar de que estos son los criterios aceptados, es preciso recordar que una población importante de sujetos con hiperaldosteronismo primario serán normokalemicos ${ }^{27,68} \mathrm{y}$, por lo tanto, este no debe ser considerado como criterio fundamental al momento de determinar qué individuo debe ser o no tamizado.

El valor de corte de ARR no es el mismo para distintos grupos de poblaciones. Debe ajustarse de acuerdo con la ingesta de sal y ser interpretado en relación con la concentración de aldosterona plasmática (debe ser $>15 \mathrm{ng}$ $\mathrm{dl}^{-1}$ ) y con la actividad de renina plasmática, que debe de estar en el límite inferior detectable. ${ }^{67}$ Además, su medición debe realizarse durante la mañana y después de 30 minutos de que el paciente haya permanecido sentado ${ }^{71}$, lo que es fundamental para no incurrir en errores de interpretación.

Se debe tomar en cuenta que los diuréticos, los bloqueadores de canales de calcio, los antagonistas de la aldosterona, los inhibidores de la enzima convertidora de angiotensina, los bloqueadores de los receptores de angiotensina II y los $\beta$-bloqueadores, en general, alteran los resultados de la prueba de tamizaje. ${ }^{69,70,72,73}$ Conviene considerar su suspensión de 2 a 3 semanas antes de tomar las pruebas de tamizaje, cuando no exista contraindicación. ${ }^{73}$ Existen algunas excepciones de drogas antihipertensivas que no alteran de forma importante los resultados ${ }^{74,75}$ y que podrían utilizarse previa realización de la prueba. La primera elección es $\alpha$-bloqueadores (como doxazosina); como segunda opción se puede usar calcio antagonistas dihidropiridínicos (como amlodipina). ${ }^{67,73} \mathrm{Si}$ el tratamiento no puede descontinuarse del todo, existe evidencia de que una ARR $>100$ (independiente de la postura y a pesar del uso de varios medicamentos antihipertensivos) es altamente

\section{Cuadro 2. Criterios para tamizaje en hiperaldosteronismo primario}

1. Hipertensión asociada a hipokalemia, ya sea "espontánea" o inducida por diuréticos ${ }^{10,12}$

2. Hipertensión resistente a tratamiento (tratamiento triple, a dosis adecuadas y que incluya un diurético) ${ }^{10,12}$

3. Incidentaloma adrenal ${ }^{10,12}$

4. Historia de hipertensión o accidente vascular cerebral en familiares inmediatos menores de 50 años $^{10}$

específica y sensible. ${ }^{76,77}$ Se debe mencionar que 2 ensayos clínicos (uno prospectivo en pacientes hipertensos esenciales $\mathrm{y}$ otro retrospectivo en pacientes con el diagnóstico de aldosteronoma) han demostrado que ni el uso de antihipertensivos ni la dieta afectan de manera significativa la precisión de la prueba. ${ }^{78.79}$

Hasta el momento, la combinación de una ARR aumentada y una concentración de aldosterona plasmática $>15 \mathrm{ng} \mathrm{dl}^{-1}$ son la mejor combinación para tamizaje. ${ }^{67}$ Sin embargo, todavía no se ha logrado responder el dilema de si el tamizaje debe de ser aún más amplio. ${ }^{80}$

Es importante enfatizar que ARR es una prueba de tamizaje y no diagnóstica y que todo resultado positivo debe de ser confirmado mediante una prueba de supresión. Los 2 test que se usan con este fin son la carga de sodio y la supresión con fludrocortisona (Cuadro 3) $6{ }^{67}$ A pesar de esto, hay quienes consideran que la combinación de $A R R>30$ en un paciente sentado, en conjunto con PAC $>15 \mathrm{ng} / \mathrm{l}$ y $\mathrm{PRA}<$ $1 \mathrm{ng} / \mathrm{ml} / \mathrm{h}$, son diagnósticos. ${ }^{12}$

Una vez hecho el diagnóstico, el siguiente paso es determinar el subtipo de hiperaldosteronismo primario, especialmente diferenciar el aldosteronoma e hiperplasia

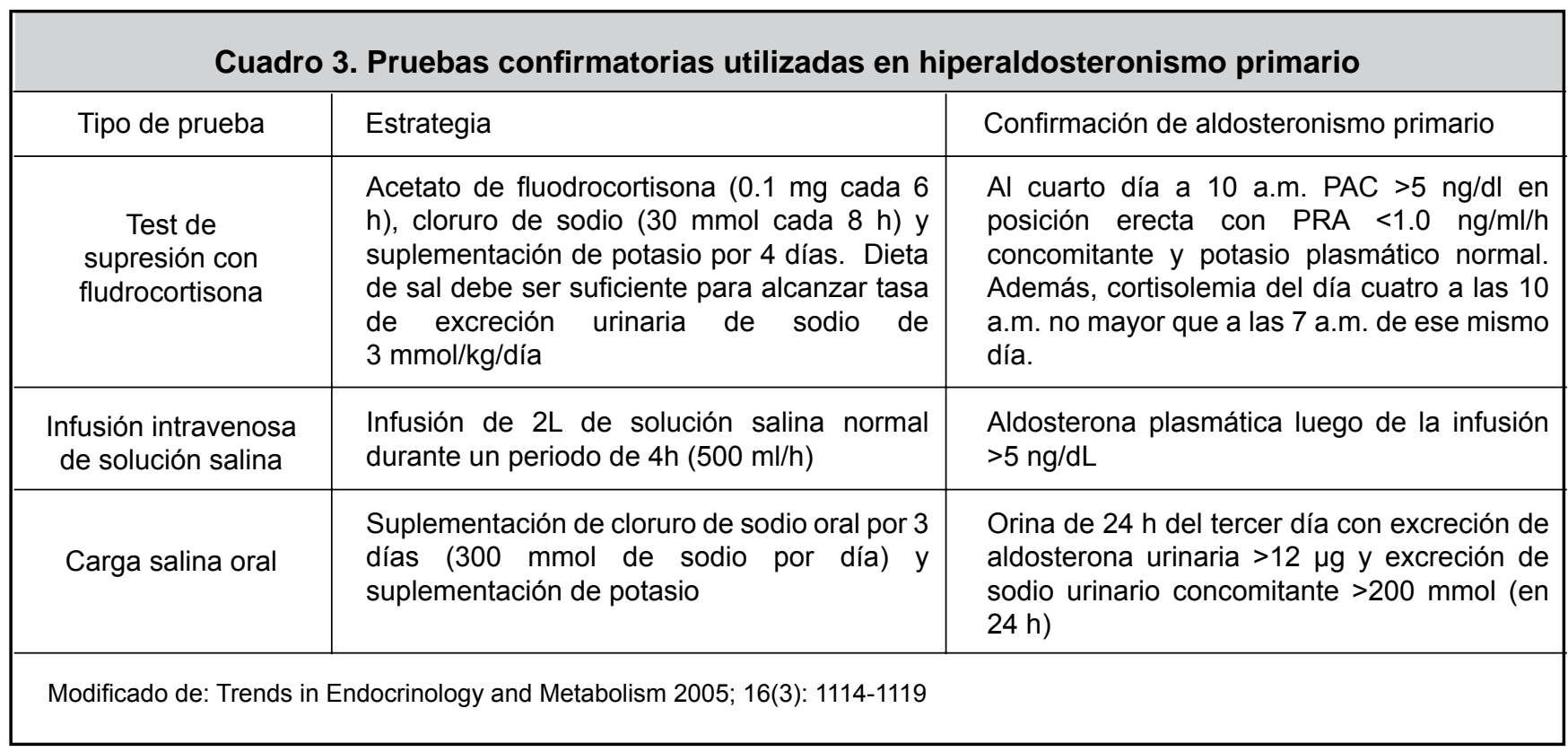


unilateral de la hiperplasia bilateral, ya que el tratamiento de estas entidades es distinto (Figura 3). El muestreo venoso adrenal es el mejor método para distinguir la hiperplasia bilateral del aldosteronoma ${ }^{81,82}$, pero requiere personal capacitado para su realización y no está disponible en muchos centros. La tomografía axial computarizada, disponible en la mayoría de centros, se puede utilizar para hacer esta distinción ${ }^{83}$, no obstante, y a pesar de que es más sensible que la resonancia magnética, el estudio tiene una sensibilidad de aproximadamente el $50 \%{ }^{67}$

El diagnóstico del aldosteronismo remediable con glucocorticoides se hace mediante una reacción en cadena de la polimerasa que se debe confirmar con análisis de Southern blot. ${ }^{84}$

\section{Manejo}

La primera pregunta que un clínico debe hacerse al tratar a un paciente es ¿por qué? En el caso del hiperaldosteronismo primario la meta de tratamiento es muy clara, ya que se trata de prevenir la morbilidad y mortalidad asociada con la hipertensión, hipokalemia y daño cardiovascular. ${ }^{10}$

\section{Tratamiento médico}

Se ha especulado que diferentes grupos de antihipertensivos podrían ser eficaces en el tratamiento del hiperaldosteronismo primario. Sin embargo, hasta el momento no existe ningún estudio controlado aleatorizado que haya comparado la eficacia relativa de estas drogas, y el tratamiento sigue siendo empírico. Lo que se ha comprobado es que el exceso de aldosterona tiene efectos deletéreos cardiovasculares independientes de la hipertensión, por lo que un antagonista de su acción debe formar parte del tratamiento. ${ }^{10}$ La droga que ha sido utilizada en este contexto es la espironolactona. La espironolactona es un inhibidor competitivo de la unión de la aldosterona a su receptor mineralocorticoide y, por lo tanto, impide la síntesis de las proteínas necesarias para llevar a cabo sus funciones. ${ }^{85,86} \mathrm{Su}$ uso está indicado en el tratamiento a largo plazo de los pacientes con hiperplasia adrenal bilateral, o en aquellos con aldosteronoma, que no desean cirugía o cuyo riesgo quirúrgico es demasiado alto. ${ }^{87}$ Además, se utiliza durante el preoperatorio de aquellos pacientes con hiperaldosteronismo primario que requieren cirugía ${ }^{87}$ En los estudios iniciales con espironolactona, las dosis utilizadas eran de hasta 400 $\mathrm{mg}$ al día, sin embargo, se sabe que dosis de hasta $100 \mathrm{mg}$ al día tienen probablemente la misma eficacia con menores efectos secundarios antiandrogénicos. ${ }^{48}$ Esta eficacia de disminución en la presión arterial es de cerca de 40-60 $\mathrm{mmHg}$ de presión sistólica y de $10-20 \mathrm{mmHg}$ de presión diastólica. $^{48} \mathrm{La}$ eplerenona no ha sido estudiada comparativamente con espironolactona en el contexto de aldosteronismo primario, por lo que no se conoce su eficacia relativa.

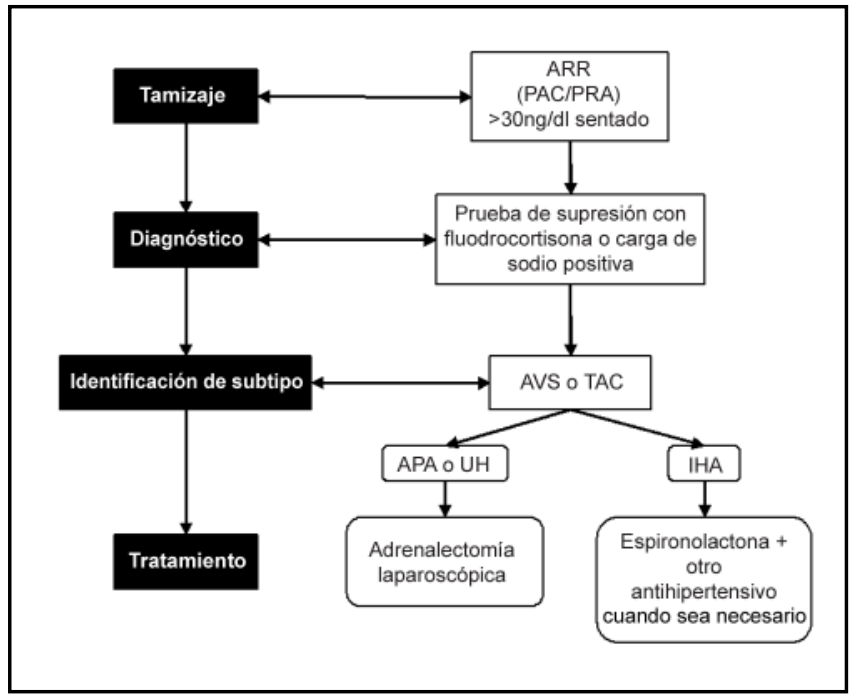

Figura 3. Algoritmo general de manejo del paciente con aldosteronismo primario

Debido a la dificultad del control de la presión arterial, solamente un $50 \%$ de los pacientes se controlan con monoterapia con espironolactona ${ }^{48}$, por lo que en muchos casos hay que agregar más agentes antihipertensivos. Hasta el momento no se sabe cuál es ese antihipertensivo ideal. Hay estudios con resultados mixtos en relación con la acción beneficiosa de los calcio antagonistas, ya que la nifedipina demostró capacidad de disminuir niveles de aldosterona como tratamiento agudo ${ }^{88}$, efecto que no fue reproducido después. ${ }^{89} \mathrm{Se}$ cree que el irbesartan podría ser beneficioso en pacientes que no han respondido a la combinación de espironolactona con calcio antagonistas. ${ }^{90}$ Tanto el triamtereno como el amiloride pueden utilizarse, sin embargo, no son primera elección de tratamiento como monoterapia, por ser menos eficaces que espironolactona ${ }^{48}$, los fármacos inhibidores de la enzima convertidora de angiotensina tampoco se ha comprobado que tengan algún beneficio adicional a su efecto antihipertensivo, excepto teóricamente en el contexto de IHA, por ser esta enfermedad dependiente de angiotensina II. ${ }^{48}$

\section{Tratamiento quirúrgico}

Durante los últimos años la laparoscopía se ha convertido en la técnica estándar de oro para la realización de adrenalectomías ${ }^{91}$, ya que es eficaz y efectiva en comparación con cirugía abierta. ${ }^{92}$ Es el tratamiento de elección para el aldosteronoma y la hiperplasia unilateral. ${ }^{93}$ Después del procedimiento quirúrgico se corrigen casi todos los casos de hipokalemia $^{12}$ y se logra la normalización de la presión arterial, sin la necesidad de uso de varios medicamentos ${ }^{12}$, pero hasta un $50 \%$ de estos pacientes continuarán siendo hipertensos. ${ }^{94}$ Un buen resultado quirúrgico depende de: corta duración de la hipertensión, ausencia de historia familiar de hipertensión, ausencia de insuficiencia renal, buena respuesta a espironolactona previo a cirugía, índice de masa ventricular izquierda normal, entre otros. ${ }^{12}$ 
Se espera que para 2025 haya un aumento en la prevalencia de la hipertensión en un $60 \%$ lo que implica que casi un $30 \%$ de la población mundial será hipertensa. ${ }^{95}$ Ante esta preocupante epidemia y la evidencia de que un porcentaje no despreciable de individuos podrían tener una causa curable, se deben tomar las medidas necesarias en la práctica médica diaria con el de tamizar y diagnosticar a aquellos pacientes con hiperaldosteronismo primario.

\section{Abstract}

Primary aldosteronism was first described fifty years ago, and until recently it was considered uncommon and an exceptional cause of hypertension. Now we know that approximately one out of every 10 patients with hypertension will have some form of hyperaldosteronism, making this group of diseases the number one cause of potentially curable hypertension. This increase in prevalence is probably due to improved screening strategies. Although, these new findings do not necessarily imply the need to screen all the patients with hypertension, it is fundamental that physicians treating hypertensive patients be familiarized with primary hyperaldosteronism so that they consider this possibility as they manage and follow this individuals patients.

\section{Referencias}

1. Williams JS, Williams GH. 50th anniversary of aldosterone. J Clin Endocrin Metab 2003; 88: 2364-2372.

2. Arlt W, Allolio B. Adrenal insufficiency. Lancet 2003; 361: 1881-93.

3. Løvås K, Husebye ES. Addison's disease. Lancet 2005; 365: 205861.

4. Simpson SA, Tait JF, Bush IE. Secretion of a salt-retaining hormone by the mammalian adrenal cortex. Lancet 1952; 2:226-228.

5. Conn JW. Presidential address: 1) Painting background. 2) Primary aldosteronism, a new clinical syndrome. J. Lab. Clin. Med. 1955; 45:3-17.

6. McDermott MT. Endocrine secrets. 4th ed., USA: Elsevier, 2005.

7. Ganguly, A. Primary aldosteronism. N Engl J Med 1998; 339:182934.

8. Don BR, Biglieri EG, Schambelan M. Hipertensión Endocrina. En: Greenspan FS, Strewler GJ, ed. Endocrinología Básica y Clínica. 4 ed., México: El Manual Moderno, 1998:411-434.

9. Vallotton MB. Primary aldosteronismo. I Diagnosis of primary aldosteronism. Clin Endocrnol. 1999;45:47-52.

10. Young WF. Minireview: Primary aldosteronism. Endocrinology 2003; 144: 2208-2213.

11. Opocher G, Rocco S, Carpene G, Armanini D, Mantero F. Minerva Endocrinol 1995;20: 49-54. (Abstract)

12. Fehaily MA, Duh QY. Clinical manifestation of aldosteronoma. Surg Clin N Am 2004; 84:887-905.
13. Stern N, Tuck ML. The adrenal cortex and mineralocorticoid hipertension. En: Lavin N, ed. Manual of Endocrinology and Metabolism. 3 ed., Philadelphia: Lippincott Williams \& Wilkins, 2002:115-144.

14. Young WF Jr. Primary aldosteronism - treatment options. Growth Horm IGF Res. 2003; 13 Suppl A: S102-8. (Abstract)

15. Montori VM, Young WF Jr. Use of plasma aldosterone concentrationto-plasma renin activity ratio as a screening test for primary aldosteronism. A systematic review of the literature. Endocrinol Metab Clin North Am 2002;31:619-32.

16. Veglio F, Morello F, Rabbia F, Leotta G, Mulatero P. Recent advances in diagnosis and treatment of primary aldosteronism. Minerva Med 2003;94:259-65. (Abstract)

17. Williams GH, Dluhy RG. Enfermedades de la corteza suprarrenal. En: Braunwald E, Isselbacher KJ, Petersdorf RG, Wilson JD, Martin JB, Fauci AS, ed. Harrison: Principios de Medicina Interna. 7ed., tomo II., Interamericana McGraw Hill, 1989:2139-2163.

18. Litchfield WR, Dluhy RG. Primary aldosteronism. Endocrinol Metab Clin North Am 1995; 24:593-612.

19. Gordon R. Mineralocorticoid hypertension. Lancet 1994; 344:240 243.

20. Nadar S, Lip G, Beevers DG. Primary hyperaldosteronism. Ann Clin Biochem 2003;40: 439-452.

21. Loh KC, Koay ES, Khaw MC, Emmanuel SC, Young Jr WF. Prevalence of primary aldosteronism among Asian hypertensive patients in Singapore. J Clin Endocrinol Metab 2000; 85:2854-2859.

22. Rossi E, Regolisti G, Negro A, Sani C, Davoli S, Perazzoli F. High prevalence of primary aldosteronism using postcaptopril plasma aldosterone to renin ratio as a screening test among Italian hypertensives. Am J Hypertens 2002; 15:896-902.

23. Rayner BL, Opie LH, Myers JE, Trinder YA, Davidson JS. Screening for primary aldosteronism normal ranges for aldosterone and renin in three South African population groups. S Afr Med J 2001; 91;594599

24. Gordon RD, Stowasser M, Tunny TJ, Klemm SA, Rutherford JC. High incidence of primary aldosteronism in 199 patients referred with hypertension. Clin Exp Pharmacol Physiol 1994; 21:315-318.

25. Kumar A, Lall SB, Ammini A, Peshin SS, Karmarkar MG, Talwar KK, Seth SD. Screening of a population of young hypertensives for primary hyperaldosteronism. J Hum Hypertens 1994;8:731-732.

26. Lim P, Dow E, Brennan G, Jung R, MacDonald TM. High prevalence of primary aldosteronism in the Tayside hypertension clinic population. J Hum Hypertens 2000; 14:311-315.

27. Fardella C, Mosso L, Gomez-Sanchez C, Cortes P, Soto J, Gomez L, Pinto M, Huete A, Oestreicher E, Foradori A, Montero J. Primary hyperaldosteronism in essential hypertensives: prevalence, biochemical profile, and molecular biology. J Clin Endocrinol Metab 200;85:1863-1867.

28. Schwatz GL. Prevalence of unrecognized primary aldosteronism in essential hypertension. American Journal of Hypertension 2002; 14 part 2:18A.

29. Schwartz GL, Turner ST. Prevalence of unrecognized primary aldosteronism in essential hypertension. Am J Hypertens 2002; 15:18A (Abstract).

30. Kreze A, Okalova D, Vanuga P, Putz Z, Kodaj J, Hrnciar J. Occurrence of primary aldosteronism in a group of ambulatory hypertensive patients. Vnitr Lek 1999;45:17-21.

31. Strauch B, Zelinka T, Hampf M, Bernhardt R, Widimsky J. Prevalence of primary hyperaldosteronism in moderate to severe hypertension in the Central Europe region. Journal of Human Hypertension 2003; 17:349-352.

32. Ganguly, A. Prevalence of Primary Aldosteronism in Unselected Hypertensive Populations: Screening and Definitive Diagnosis. The Journal of Clinical Endocrinology \& Metabolism 2001; 86:40004005 . 


\section{Hiperaldosteronismo primario / Gómez-Hernández y Chen-Ku}

33. Connell JMC. Is there an epidemic of primary aldosteronismo? Journal of Human Hypertension 2002 16, 151-152.

34. Padfield PL. Primary aldosteronism, a common entity? the myth persists. Journal of Human Hypertension 2002;16:159-162.

35. White, PC. Disorders of Aldosterone Biosynthesis and Action. N Engl J Med 1994;331: 250-258.

36. Schenermann J. B., Sayegh S.I. Kidney Physiology. U.S.A.: Lippincott-Raven, 1998.

37. Rodríguez FJ, López-Vidriero E. Mineralocorticoides, Hiperaldosteronismos, Hipoaldosteronismos. En: Jara A, ed. Endocrinología. Madrid: Médica Panamericana, 2001:243-249.

38. Beevers G, Lip G, O'Brien E. ABC of hypertension The pathophysiology of hypertension. British Medical Journal 2001; 322:1912-16.

39. Weber, K. Aldosterone in congestive heart failure. N Engl J Med; 345:1689-97.

40. Guyton AC, Hall JE. Texbook of medical physiology. 10ed., U.S.A.: W.B. Saunders Company, 2000.

41. Berne RM, Levy MN. Fisiología. 3 ed., Madrid: Harcourt, 2001.

42. Valtin H., Schafer J.A. Renal function: Mechanisms preserving fluid and solute balance in health. 3ed., U.S.A.: Little, Brown and Company, 1995.

43. Nadar S, Lip G, Beevers DG. Primary hyperaldosteronism. Ann Clin Biochem 2003;40: 439-452.

44. Onusko E. Diagnosing secondary hypertension. Am Fam Physician 2003; 67: 67-74.

45. Gill JR, Bartter FC. Overproduction of sodium retaining steroids by the zona glomerulosa is adrenocorticotropin dependent and mediates hypertension in dexametasone suppressible aldosteronism. J Clin Endocrinol Metab 1981; 53: 331-337.

46. Luft, FC. Mendellian forms of hypertension and mechanisms of disease. Clinical Medicine \& Research 2003;1:291-300.

47. Dluhy RG, Lifton RP. Glucocorticoid remediable aldosteronismo. J Clin Endocrinol Metab 1999;84:4341-44.

48. Lim PO, Young WF, McDonald T. A review of the medical treatment of primary aldosteronismo. J Hypertens 2001;19:353-361.

49. Blumenfeld JD, Sealey JE, Schlussel Y, Vaughan D, Sos TA, Atlas SA, et al. Diagnosis and treatment of primary hyperaldosteronism. Ann. Intern. Med. 1994; 121:877-885.

50. Tanabe A, Naruse M, Naruse K, Hase M, Yoshimoto T, Tanaka M, Seki T, Demura R, Demura H. Left ventricular hypertrophy is more prominent in patients with primary aldosteronism than in patients with other type of secondary hypertension. Hypertension Res 1997; 20:8590. (Abstract)

51. Nishimura M, Uzu T, Fujii T, Kuroda S, Nakamura S, Inenaga T, Kimura G. Cardiovascular complications in patients with primary aldosteronism. Am J Kidney Dis 33:261-266

52. Rocha, R, Funder JW. The pathophysiology of aldosterone in the cardiovascular system. Ann. N. Y. Acad. Sci 2002; 970, 89-100. (Abstract)

53. Rossi GP, Sacchetto A, Pavan E, Palatini P, Graniero GR, Canali C, Pessina A. Remodeling of the left ventricle in primary aldosteronism due to Conn's adenoma. Circulation 1997; 95:1471-1478.

54. Rossi GP, Di Bello V, Ganzaroli C, Sacchetto A, Cesari M, Bertini A et al. Excess aldosterone is associated with alterations of myocardial texture in primary aldosteronism. Hypertension 2002;40:23-27.

55. McMahon GT, Dluhy RG. Glucocorticoid-remediable aldosteronism. Cardiol. Rev 2004; 12:44-48.

56. Struthers AD. Aldosterone-induced vasculopathy. Mol. Cell. Endocrinol 2004;217:239-241.

57. Young Jr WF. Primary aldosteronism: update on diagnosis and treatment. Endocrinologist 1997;7:213-221.
58. Gennari FJ. Hypokalemia. N Engl J Med 1998; 339:351-58.

59. Cotran RS, Kumar V, Collins T. Patología structural y functional. 6ed., Mexico: McGraw-Hill Interamericana, 2000.

60. Ulick Stanley, Blumenfeld JD, Atlas SA, Wang JZ, Vaughan ED. The unique steroidogenesis of th aldosteronoma in the differential diagnosis of primary aldosteronismo. J Clin Endocrin Metab 1993; 76:873-878.

61. Stowasser M, Huggard PR, Rossetti TR, Bachmann AW, Gordon RD. Biochemical evidence of aldosterona overproduction and abnormal regulationinnormotensiveindividualswithfamilialhyperaldosteronism type I. J Clin Endocrin Metab 1999;84: 4031-4036

62. Hiramatsu K, Yamada T, Yukimura Y, et al. A screening test to identify aldosterona producing adenoma by measuring plasma renin activity: results in hypertensive patients. Arch Intern Med 1981;141:1589-93.

63. Montori VM, Young Jr WF 2002 Use of plasma aldosterone concentrationto- plasma renin activity ratio as a screening test for primary aldosteronism. A systematic review of the literature. Endocrinol Metab Clin North Am 31:619-632, xi 15

64. Bravo EL. Primary aldosteronism. Issues in diagnosis and management. Endocrinol Metab Clin North Am 1994; 23:271-283.

65. Grim CE. Evolution of diagnostic criteria for primary aldosteronism: why is it more common in "drug-resistant" hypertension today? Curr Hypertens Rep 2004; 6: 485-92. (Abstract)

66. Mulatero, P. et al. Increased diagnosis of primary aldosteronism, including surgically correctable forms, in centers from five continents. J. Clin. Endocrinol. Metab. 2004; 89:1045-1050.

67. Mulatero P, Dluhy RG, Giacchetti G, Boscaro M, Veglio F, Stewart PM. Diagnosis of primary aldosteronism: from screening to subtype differentiation. Trends in Endocrinology and Metabolism 2005; 16:1114-1119.

68. Gordon RD. Primary aldosteronism. J Endocrinol Invest 1995; 18:495-511.

69. Buhler FR, Laragh JH, Baer L, Vaughan ED, Brunner HR. Propanol inhibition of rennin secretion: a specific approach to diagnosis and treatment of rennin-dependent hypertensive diseases. N Engl J Med. 1972; 287: 1209-1214.

70. Gordon MS, Williams GH, Hollenberg NK. Renal and adrenal responsiveness to angiotensin II: influence of $\beta$-adrenergic blockade. Endocr Res. 1992;18: 115-131.

71. Tiu SC, Choi CH, Shek CC, Ng YW, Chan FK, Ng CM, Kong AP. The Use of Aldosterone-Renin Ratio as a Diagnostic Test for Primary Hyperaldosteronism and Its Test Characteristics under Different Conditions of Blood Sampling. The Journal of Clinical Endocrinology \& Metabolism 2005; 90:72-78.

72. Brown MJ, Hopper RV. Calcium-channel blockade can mask the diagnosis of Conn's syndrome. Postgrad Med J. 1999;75:235-236.

73. Mulatero, P. et al. Drug effects on aldosterone/plasma renin activity ratio in primary aldosteronism. Hypertension 2002;40:897-902.

74. Young WF Jr. Primary aldosteronism: a common and curable form of hypertension. Cardiol Rev. 1999;7:207-214.

75. Oliveros-Palacios MC, Godoy-Godoy N, Colina-Chourio JA. Effects of doxazosin on blood pressure, rennin-angiotensin-aldosterone and urinary kallikrein. Am J Cardiol. 1991;67:157-161.

76. Gallay BJ, Ahmad S, Xu L, Toivola B, Davidson RC. Screening for primary aldosteronism without discontinuing hypertensive medications: plasma aldosterone renin ratio. Am J Kidney Dis. 2001;37:699-705.

77. Lei Xu, Viering E, Davidson RC, Toivola B: The diagnosis of primary hyperaldosteronism (PHA) using plasma aldosterone-to-renin activity ratio. Am J Clin Pathol 1994;102:257A. (Abstract)

78. Schawartz GL, Turner ST. Screening for primary aldosteronism in essential hypertension: diagnostic accuracy of the ratio of plasma aldosterone concentration to plasma renin activity. Clin Chem 2005; 51:386-94. 
79. Tanabe A, Naruse M, Takagi S, Imaki T, Takano K. Variability in the Renin/Aldosterone Profile under Random and Standardized Sampling Conditions in Primary Aldosteronism. J Clin Endocrinol Metab 88:2489-2494.

80. Francois $\mathrm{P}$, Jeunemaitre $\mathrm{X}$. Would wider screening for primary aldosteronism give any health benefits? European Journal of Endocrinology 2004; 151:305-308.

81. Stowasser M, Gordon RD, Rutherford JC, Nikwan NZ, Daunt N, Slater GJ. Diagnosis and management of primary aldosteronism. JRAAA 2001;2:156-169.

82. Magill SB, Raff H, Shaker JL, Brickner RC, Knechtges TE, Kehoe ME, Findling JW. Comparison of adrenal vein sampling and computed tomography in the differentiation of primary aldosteronismo. J Clin Endocrinol Metab 2001;86:1066-1071.

83. White EA, Schambelan M, Rost CR, Biglieri EG, Moss AA, Korobkin M. Use of computed tomography in distinguishing the cause of primary aldosterism. N Engl J Med 1980; 303;1503-07.

84. Mulatero P, Morello F, Veglio F. Genetics of primary aldosteronism. J Hyperten 2004; 22: 663-670.

85. Jackson EK. Diuretics. En: Hardman JG, Limbird LE, editors. Goodman and Gilman's the pharmacological basis of therapeutics. 10th ed. New York: McGraw-Hill;2001:757-787.

86. Flórez J, Armijo JA. Fármacos diuréticos. En: Flórez J, editor. Farmacología humana. 3 ed. Barcelona: Masson; 1997:815-829.

87. Mosby's Drug Consult. www.mdconsult.com. Sitio accesado 15 de noviembre del 2005
88. Nadler JL, Hsueh W, Horton R. Therapeutic effect of calcium channel blockade in primary aldosteronism. J Clin Endocrin Metab 1985;60:896-899.

89. Carpene G, Rocco S, Opacher G, Mantero F. Acute and chronic effect of nifedipine in primary aldosteronism. Clin Exp Hypertens 1989;11:1269-1272. (Abstract)

90. Stokes GS. Monaghan JC, Ryan M, Woodward M. Efficacy of an angiotensin II receptor antagonist in managing hyperaldosteronism. J Hypertens 2001;19:1161-65.

91. Smith DC, Weber CJ, Amerson JR. Laparoscopic Adrenalectomy: New Gold Standard. World J. Surg. 1999;23:389-396.

92. Shen WT, Lim RC, Siperstein AE, et al. Laparoscopic vs open adrenalectomy for the treatment of primary hyperaldosteronism. Arch Surg 1999; 134:628-32.

93. Chavez J, Pasieka JL. Adrenal lesions assessed in the era of laparoscopic adrenalectomy: a modern day series. The American Journal of Surgery 2005;189: 581-586.

94. Plouin PF, Amar L, Chatellier G. Trends in the prevalence of primary aldosteronism, aldosterone producing adenomas, and surgically correctable aldosterone dependent hypertension. Nephrology, Dialysis, Transplantation 2004;19:774-777.

95. Kearney PM, Whelton M, Reynolds K, Muntner P, Whelton PK, He J. Global burden of hypertension: analysis of worldwide data. Lancet 2005;365:217-223.

\section{Costa Rica en el exterior}

\section{Relación entre el tipo de papilomavirus humano, carga viral cualitativa y la edad con anormalidad citológica.}

Kovacic MB, Castle PE, Herrero R, Schiffman M, Sherman ME, Wacholder S, Rodriguez AC, Hutchinson ML, Bratti MC, Hildesheim A, Morales J, Alfaro M, Burk RD.

Cancer Res. 2006 Oct 15;66(20):10112-9. Infecciones cervicales persistentes con papilomavirus humanos (HPV) carcinogénicos causan prácticamente todos los cánceres cervicales. Las anormalidades citológicas son las manifestaciones de las infecciones por HPV utilizadas para identificar las mujeres en riesgo. Para comparar el potencial del espectro completo de los genotipos de HPV anogenitales para inducir efectos citopatológicos, examinamos la influencia del tipo de HPV, la carga viral y la edad sobre la citopatología entre 1222 mujeres, con un solo tipo de HPV, en el momento de reclutamiento, en un estudio basado en una población de 10000 mujeres en Costa Rica. Muestras cervicales fueron analizadas para aproximadamente 40 tipos de HPV con PCR utilizando los primer MY09/MY11 L1 e hibridación dot blot tipo específica. Los tipos fueron organizados por especies filogenéticas y riesgo de producir cancer. La intensidad de la banda del PCR sirve como un indicador cualitativo de la carga viral. En conjunto, 24.8\% [95\% intervalo de confianza (95\% IC), 22.4-27.3] de infecciones únicas prevalentes por HPV tuvieron, al mismo tiempo, anormalidades (células planas atípicas o peor) con un rango desde $0.0 \%$ hasta $80.0 \%$ basados en el tipo de HPV. Los tipos alfa3/alfa15 no carcinogénicos, a pesar de ser altamente prevalentes, rara vez causaron anormalidades citológicas (13.1\%; 95\% IC, 9.8-17.0). En contraste, de un cuarto a casi la mitad de las infecciones con un único tipo de especie muy carcinogénica(alfa9/alfa11/alfa7/alfa5/ alfa6) produjeron anormalidades. Mayores anormalidades fueron observadas con cargas virales cualitativamente crecientes de tipos carcinogénicos; menos anormalidades fueron observadas entre mujeres mayores ( $>54$ años). Un alto porcentaje $(46,2 \%)$ de las anormalidades detectadas en mujeres infectadas con HPV16 o con los tipos alfa9 relacionados fueron grado severo o peor, consistente con una fuerte carcinogenicidad, comparado con $10.7 \%$ de mujeres infectadas con tipos alfa 7, incluyendo HPV18, una causa importante de adenocarcinoma. La falta de anormalidades severas evidentes asociadas con HPV18 y tipos HPV relacionados tiene implicaciones en el tamizaje de lesiones glandulares y lesiones relacionadas con el tipo alfa7. 